\title{
Differences in Developing the Lever-pressing Behavior in Two Strains of Rats
}

\author{
TIMOTHY O. T. TS'O AND SANDRA J. BAKER \\ DIG Chemical Biology Research, The Dow Chemical Company, Midland MI 48640
}

AND

NANCY A. NOBLE AND GEORGE J. BREWER

Department of Human Genetics, University of Michigan, Ann Arbor MI 48104

(Received 19 July 1974)

\begin{abstract}
TS'O, T. O. T., S. J. BAKER, N. A. NOBLE AND G. J. BREWER. Differences in developing the lever-pressing behavior in two strains of rats. PHYSIOL. BEHAV. 14(6) 719-723, 1975. - Learning to lever-press for food in rats may be experimentally analyzed according to (a) association of lever-pressing with food reinforcement; (b) development of the behavior of lever-pressing. The latter was studied in two strains of rats with a high or low level of 2,3-diphosphoglycerate (DPG) in ery throcytes. Twelve males from each strain, previously trained to lever-press, were used. The rate of responding increased faster with repeated training-testing sessions in the High Strain than in the Low Strain under the CRF and the FR 10 schedule. The genetic difference in the DPG level in erythrocytes and the resultant long term difference in oxygen transport capability may be a significant factor contributing to the behavioral differences in these two strains.
\end{abstract}

Development of lever-pressing behavior Genetic influence Erythrocy te 2,3-diphosphoglycerate level Strain differences

THE association between a stimulus and the proper response in a problem solving situation is the initial part of the process of learning, and was summarized by Thorndike's Law of Effect [11]. On the other hand, in the leverpressing operant situation, the animal has to develop not only the association of lever-pressing with the delivery of food but also the proficiency of the act of lever-pressing, an act not a part of the animal's natural behavioral repertoire. Such proficiency is a continuous variable which depends on the amount of experience in the problem solving situation and can be measured by the rate of lever-pressing. This particular aspect of the learning process can only be studied by placing a strict experimental control on the initial conditions of the association of lever-pressing with food reinforcement. The availability of a computer-controlled behavioral system and a rapid, automatic procedure to train a large number of animals provided such an opportunity [ 8 , $9,10]$. The presence of two strains of rats genetically selected according to a biochemical marker provided another opportunity [7]. The present study analyzed and compared the development of the lever-pressing skill in these two strains of rats.

The criterion of the selective breeding process is the level of 2,3-diphosphoglycerate (DPG), a glycolytic intermediate of carbohydrate metabolism, in the red blood cells. The level of DPG in red blood cells is considered to be an important controlling factor in the oxygen transport to tis- sues, because the binding of DPG to the hemoglobin molecules reduces the affinity of hemoglobin for oxygen [2, 3 , 4]. Thus a higher level of DPG in a standard concentration of hemoglobin in blood would result in a larger amount of oxygen delivered to the tissues of the whole body without an increase in cardiac output or a decrease in tissue capillary oxygen pressure. The Long-Evans strain of rats was chosen as the experimental animal model because it has the same degree of natural variability in DPG level as man, although the absolute levels are higher. In the selective breeding process, the animals with the highest levels and the lowest levels of DPG were mated among themselves. After 3 generations, the DPG level did not increase further in the High strain or decrease further in the Low strain. The High strain then had different oxygen dissociation curves displaced to the right of those of the Low strain. At p50, there was a $6 \mathrm{~mm} \mathrm{Hg}$. pressure difference, an indication of a functional difference between the two strains in their oxygen transport [3]. The higher DPG level in the High strain was believed to be causally related to the higher activity of the enzyme phosphofructokinase [7].

\section{METHOD}

\section{Animals}

Two strains of Long-Evans rats were selectively bred for a high and low level of erythrocyte 2,3-diphosphosglycerate 
at the Department of Human Genetics, University of Michigan, Ann Arbor, Michigan [7]. These rats were originally obtained from Simonsen Laboratories, California, and were bred by mating the animals with the highest level and the lowest level among themselves. Twelve male rats, aged $122-134$ days from the 10 th generation from each strain were used. Estimates of inbreeding in each strain were calculated according to the method of Falconer [5]. The inbreeding coefficient for the 10 th generation of the High strain is 0.32 and that for the Low strain is 0.28 .

All 24 rats were trained to lever-press for food pellets in three 15 hr overnight sessions, using a computer-controlled behavioral system $[8,9,10]$. The 24 rats were selected from a group of 36 animals (18 from each strain) because they had a very similar initial learning experience. The criteria for their selection were as follows: (1) All animals had associated the act of lever-pressing with food reinforcement. (2) All animals had lever-pressing experience on Fixed Ratio schedules, namely Continuous Reinforcement, FR 2, FR 5, and FR 10 schedules during their three overnight training sessions. (3) All had obtained more than 100 pellets on the third session. (4) The number of pellets obtained by these two strains did not differ significantly on all three training sessions.

The free-feeding body weights of the 12 animals of the High and the Low strain were not significantly different (mean $\pm \mathrm{S}$. D. $=449 \pm 41 \mathrm{~g}$ and $416 \pm 47 \mathrm{~g}$ respectively). No remarkable differences were detected on physical examination among all these rats, both within and between groups, in regard to their general health, development and activity. All the rats of the High strain had DPG levels higher than those of the Low strain (mean \pm S. D. $=32.96 \pm$ 1.47 and $22.92 \pm 1.64$ micromoles $/ g$ of hemoglobin respectively).

\section{Apparatus}

The computer-controlled behavioral system was used $[8,10]$. Testing was done in 6 identical behavioral chambers supplied by Lehigh Valley Electronics, Inc. They are LVE 1417 Test Chambers, including $1417 \mathrm{c}$ cubicles, 1417 Test Cages each with a modified intelligence panel Type I, two $1405 \mathrm{R}$ retractable levers (activated by $23-25 \mathrm{~g}$ static weight, only the right lever was used), one 1548 Pellet Feeder (45 mg Noyes food pellets were used). A GrasonStadler 901B noise Generator supplied a low level of masking noise. An Esterline Angus 20 channel recorder, and 6 cumulative recorders were also used. The behavioral chambers were connected to an interface panel which in turn was connected to a PDP-8/i computer with an unique Foreground/Background software system. This Foreground function is devoted solely to controlling the behavioral chambers and to recording the data.

\section{Procedure}

Both groups of rats were food deprived for $24 \mathrm{hr}$ before they were introduced into the behavioral chamber for this series of experiments. This series consists of 3 experiments performed consecutively for 21 days. In the first experiment, the rats were tested with a 20 minute continuous reinforcement schedule (CRF) daily for 4 days. All animals were supplemented equally with about $7 \mathrm{~g}$ of Purina Rat Chow 3 hr after they were tested.

The second experiment lasted 5 days, namely from Day
5 to Day 9, during which the animals were tested with a 20 min Fixed Ratio 10 schedule (FR 10) daily.

The third experiment was a dose response study of intraperitoneal injections of d-amphetamine sulphate $(3,6,9$ micromoles $/ \mathrm{kg}$ i.e. $0.55,1.1,1.65 \mathrm{mg} / \mathrm{kg}$ ). This experiment lasted 11 days, namely from Day 10 to Day 21 . The same FR 10 schedule as in the second experiment was used. Saline injections were given one day before the amphetamine injections as control on Day 11,15 , and 20.

From Day 5 onward, the animals were supplemented with about $12 \mathrm{~g}$ of Purina Rat Chow daily to maintain their body weight at about $80-85$ percent of their free-feeding body weight. They were weighed daily before the test. Water was always available except in the testing chambers.

The rates of improvement of the lever-pressing skill within each strain and between the two strains as they were repeatedly exposed to the training-testing sessions were analyzed statistically. The procedure used was analysis of variance with correction for repeated measures [12], i.e. the source of variance due to repeated sampling of the same individuals was corrected for in the computation of the $F$ ratio.

\section{RESULTS}

In the first experiment, both strains showed significant increases in their respective rates of responding with repeated daily training-testing sessions (Fig. $1 ; F_{3,66}=63.7$, $p<0.001$ ). This phenomenon in both strains may be interpreted as a kind of learning process or a development of the proficiency of their lever-pressing skill. When the two strains were compared, they had an identical rate of responding on Day 1 . However, in the subsequent 3 days, the High strain developed their lever-pressing behavior at a significantly faster rate than the Low strain (Fig. $1 ; \mathrm{F}_{3,66}=$ $5.26, p<0.005$ )

In the second experiment, when first exposed to the FR 10 schedule on Day 5, both strains had quite similar initial rates of responding. Again both strains significantly increased their respective rates of responding with repeated daily training-testing sessions, indicating that they were developing their lever-pressing rate further under this new and more demanding situation (Fig. 2, $F_{4,88}=45.4$, $p<0.001$ ). When the performance of the two strains was compared, the increase of the rate of responding across days in the High strain was again significantly higher than that in the Low strain (Fig. $2, \mathrm{~F}_{4,88}=1.86, p<0.05$ ).

In the third experiment, the mean rates of responding of the animals when given the saline injections were significantly different (Fig. 3, $t=3.69, p<0.001$; the High strain had a higher rate). However, their reactions to the treatment with d-amphetamine were quantitatively and qualitatively quite similar. A biphasic dose-response curve was demonstrated (Fig. 4), i.e. an increase of the rate of responding was observed at the low dose and a decrease of the rate of responding at the higher dose. Quantitatively, at the same dose level, the Low strain reacted slightly more to the stimulant action, and the High strain more to the depressant action. These differences were not statistically significant.

\section{DISCUSSION}

In a recent comprehensive review, Bitterman and Schoel [1] distinguished two main categories of instrumental 
T47-1003

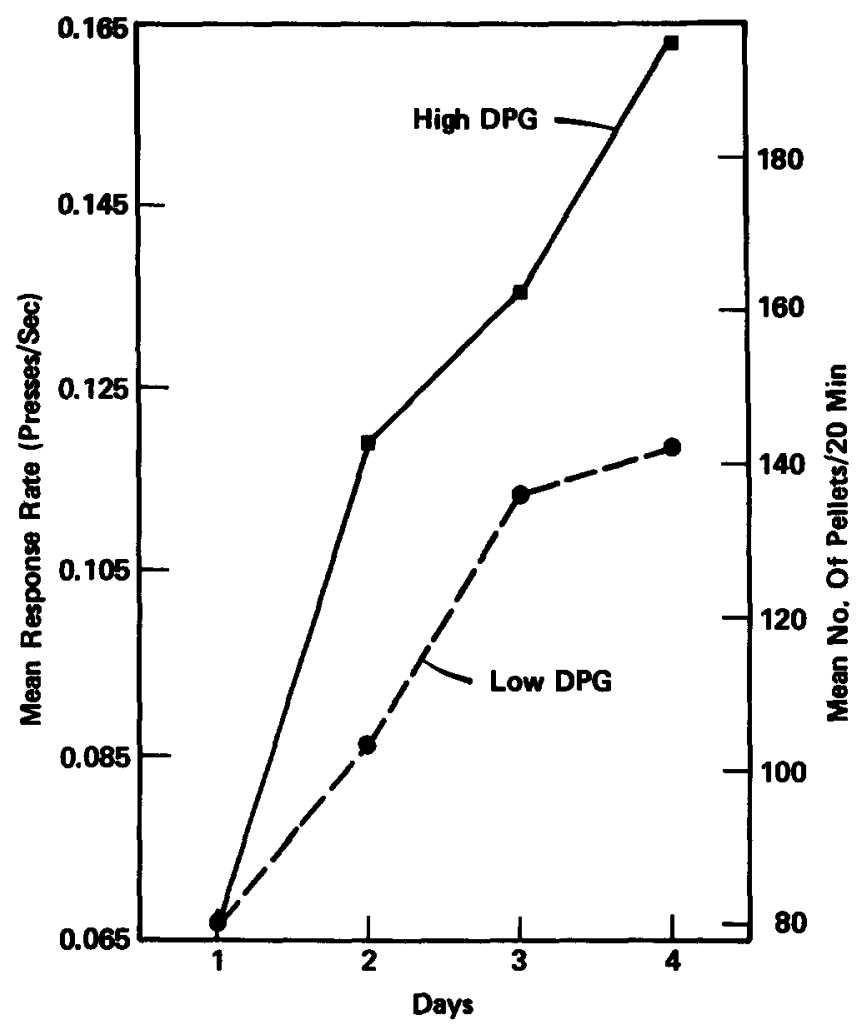

FIG. 1. The first experiment: mean response rate and mean no. of pellets obtained by the High and Low strains during a $20 \mathrm{~min}$ CRF schedule.

T47-1206

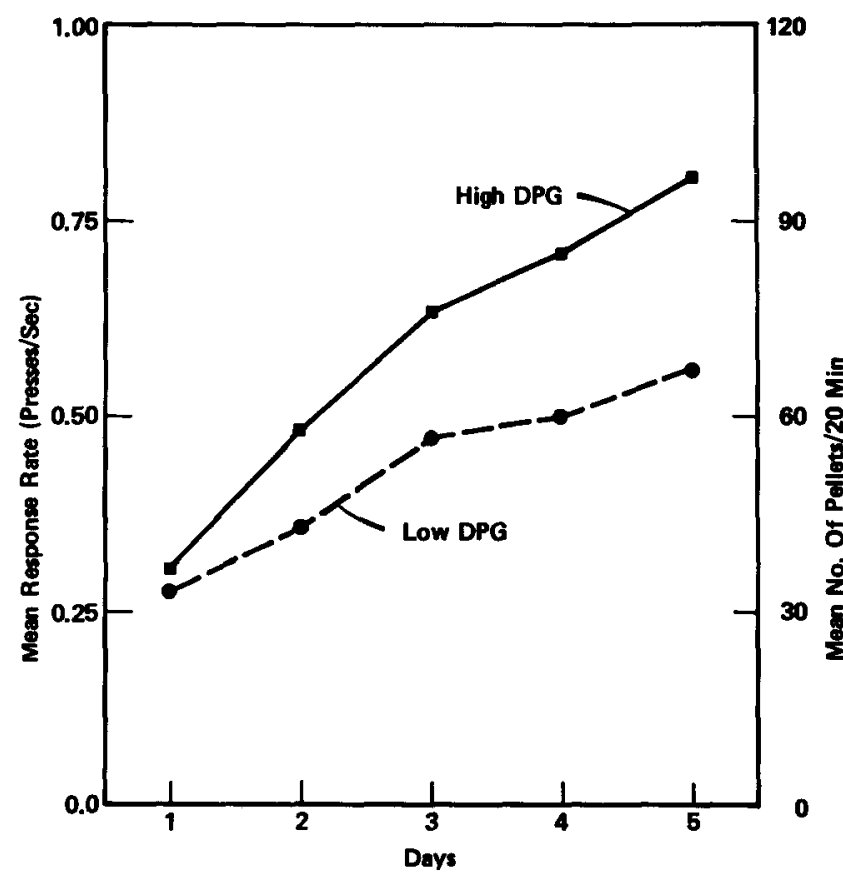

FIG. 2. Second experiment: mean response rate and mean no. of pellets obtained by the High and Low strains during a 20 min FR 10 schedule.
T47-1206

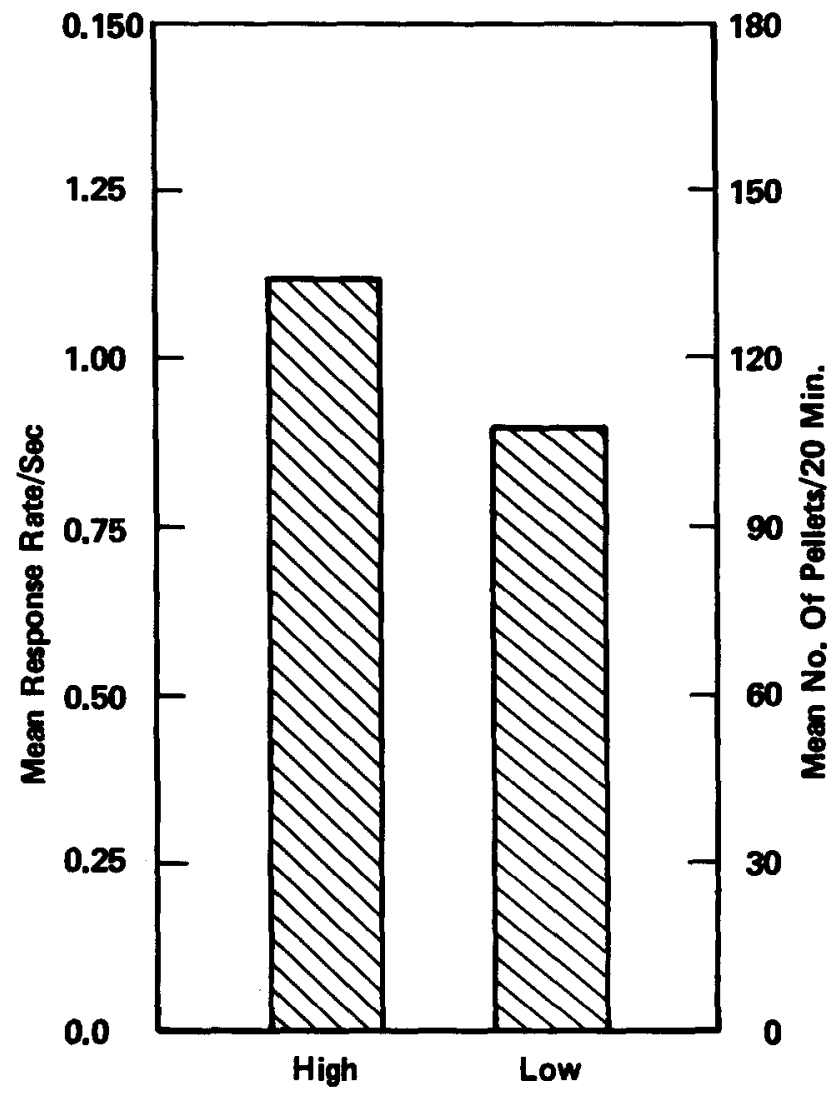

FIG. 3. The third experiment: mean response rate and mean no. of pellets obtained by the High and Low strains during a 20 min FR 10 schedule. Each bar represents the average of 3 saline injection days.

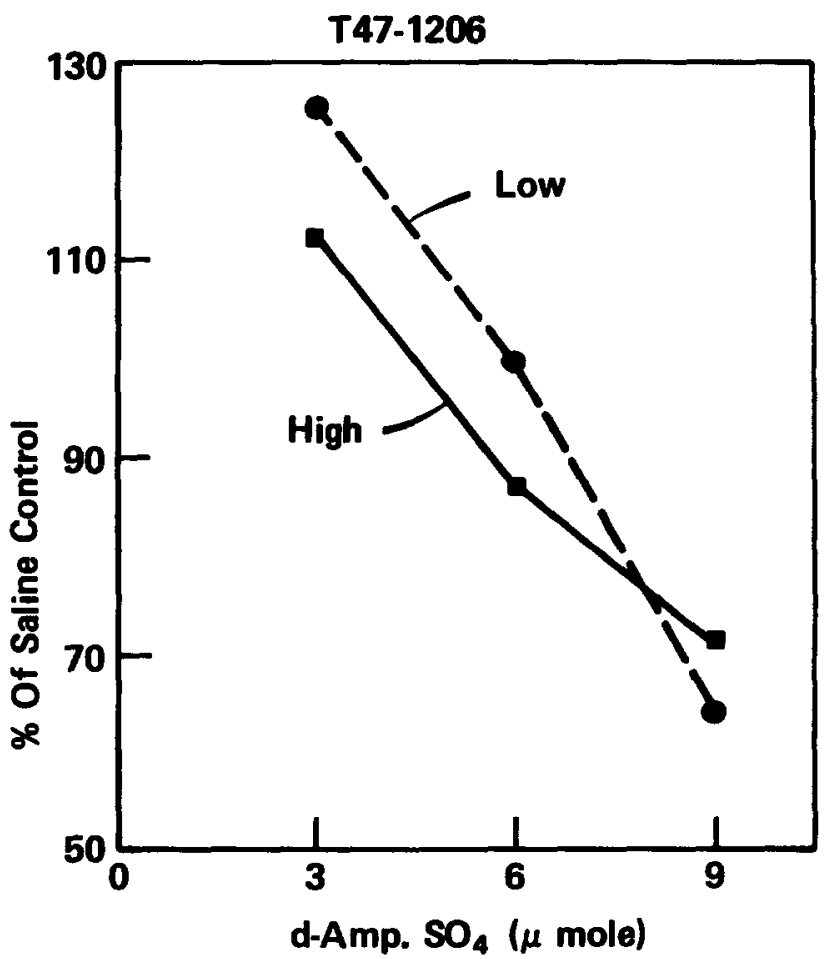

FIG. 4. The third experiment: the dose-response curves of the High and Low strains to injections of d-amphetamine sulfate. 
learning: the Thorndikian case and the avoidance case. The lever-pressing behavior we studied here belongs to the T-1 sub-category of the Thorndikian case. Our experiments further demonstrated that the learning process can be separated into two parts. The first part is the association of lever-pressing with food pellet delivery. The second part is the development of the lever-pressing rate which is being studied in this series of experiments. The difficulty in studying this process resides in the problem of baseline. The development of the lever-pressing skill cannot be evaluated unless the animals are comparable in the performance of the act of lever-pressing and in the association of such an act with food reinforcement. In these experiments, the animals were treated identically and impartially in their initial learning sessions. They were then further selected according to very stringent criteria to ensure the similarity of their performance in their initial learning of the association of lever-pressing with food pellets. The identical mean rates of responding of the two strains in the first day of the first experiment justify the rigorous selection criteria.

Therefore these experiments demonstrated that the lever-pressing skill can be studied in single individuals and can be compared between groups of animals as this skill was developed towards an asymptotic level. When the length of the training-testing session was reduced from $15 \mathrm{hr}$ to 20 min, making the amount of reinforcement contingent upon the response rate the rate of lever-pressing for food pellets improved with repeated sessions. Similarly, the rate of responding increased further when the ratio of responding to reinforcement was increased from 1 to 10 . Thus, the significant daily increase of the response rates of each strain clearly demonstrated the unique feature of the process of the development of the lever-pressing proficiency. These findings also emphasize the significant correlation between the increase of the response rate and the length and the demand of the schedule.

An equally significant finding is the behavioral differences between these two strains of rats. Under both the first and the second experimental conditions, both strains had similar initial rates of responding. However, on the second day of both experiments, it became quite obvious that the High strain was developing the lever-pressing skill faster than the Low strain. The Low strain was capable of increasing its rate of responding to certain levels equal to or higher than those attained by the High strain, except that the Low strain required more sessions of training to attain such a level. For example, in the first experiment, the rate of responding of the Low strain on Day 4 was the same as that of the High strain on Day 2. Similarly in the second experiment, under the more demanding schedule of FR 10 , the Low strain lever-pressed and obtained more pellets on the fourth and fifth day than the High strain on the first day.

Furthermore, the response rate of the Low strain on Day 5 , the first day of the second experiment, was higher than the response rate of the High strain on Day 4, the last and the highest scoring day of the first experiment (Figs. 1 and 2). Similarly, the average response rate of the Low strain during the saline injection days was higher than the response rate of the High strain on Day 9 (Figs. 2 and 3). In the last comparison, both strains were lever-pressing under the FR 10 schedule. These findings clearly show that the lower performance of the Low strain during the first and the second experiment was not due to physical incapacity or motivational differences.

In the third experiment, the response of these two strains to the central nervous system stimulant, d-amphetamine, was quite similar, but the rates of responding on the saline days were significantly different. Whether the rates of responding would eventually become equal with prolonged training cannot be ascertained at present.

Three other points should be noted. First, these two strains were selectively bred to represent the two extremes of the natural distribution of the DPG level in the population of the Long-Evans rats. Neither strain should be considered as abnormal mutants nor were they physiologically inadequate. Secondly, these rats were raised under normal laboratory conditions, and were never subjected to anoxia or other known environmental stresses. Thirdly, there is still much individual behavioral difference among all the rats and within each strain, which did not correlate with their DPG level. That is, a rat with a low DPG level in the Low strain could develop the lever-pressing skill faster than another rat with a higher DPG level regardless of which strain it belonged to. Thus the DPG level was at best only a contributory factor and not an absolute predictor of the behavioral performance of the rats.

However, the High strain, which has a better oxygen transport mechanism in its blood, is also the strain which learned faster. It is very possible that this biochemical change in oxygen delivery is one of the contributory factors to the behavioral differences observed in our experiments. This hereditary difference in one biochemical marker may have affected the development and function of the nervous system through the chronic and subtle influence of having more oxygen available to the nervous tissues from the time of conception through the time of learning and performance. Further genetic analysis by cross-breeding experiments needs to be done to demonstrate more definitively the causal relationship between this biochemical difference and the behavioral difference of these two strains.

In summary, this study demonstrated that the rate of the development of the lever-pressing rate can be measured by short sessions of CRF and FR 10 schedules. The strain of rats with higher level of erythrocyte DPG was found to develop the lever-pressing skill faster than the strain with a lower level of DPG. Furthermore, this learning process was found to be different among individual rats. The behavioral differences between the two strains may be due to the different erythrocyte DPG levels resulting in different amounts of oxygen delivered to the brains cells at the same oxygen tension.

However, the individual differences among the rats also clearly showed that the influence of the DPG level is only one of many factors inherent in each animal which would affect this process of learning.

\section{REFERENCES}

1. Bitterman, M. E. and W. M. Schoel. Instrumental learning in animals: parameters of reinforcement, A. Rev. Psychol. 21: $367-436,1970$.
2. Brewer, G. J. and J. W. Eaton. Erythrocyte metabolism: interaction with oxygen transport. Science 171: 1205-1211, 1971. 
3. Brewer, G. J., F. J. Oelshlegel, Jr. and J. W. Eaton. Biochemical, Physiological and Genetic Factors in the Regulation of Mammalian Erythrocyte Metabolism and DPG Levels. In: Oxygen Affinity of Hemoglobin and Red Cell Acid Base Status. Alfred Benzon Symposium IV, edited by $M$. Rorth and P. Astrup. New York: Academic Press, 1972, pp. 629-645.

4. Duhm, J. 2,3-DPG-induced displacements of the oxyhemoglobin dissociation curve of blood: mechanisms and consequences. In: Oxygen Transport to Tissue, Instrumentation, Methods, and Physiology, edited by H. I. Bicher and D. F. Bruley. New York: Plenum Press, 1973, pp. 179-186.

5. Falconer, E. L. Inbreeding. In: Quantitative Genetics. New York: Ronald Press, 1960, pp. 60-67.

6. Ferster, C. B. and B. F. Skinner. Schedules of Reinforcement. Appleton-Century-Crofts, Inc., New York, 1957.

7. Noble, N. A. and G. J. Brewer. Studies of the metabolic basis of the ATP-DPG differences in genetically selected high and low ATP-DPG rat strains. In: Hemoglobin and Red Cell Structure and Function, edited by G. J. Brewer. New York: Plenum Press, 1972, pp. 155-164.
8. Stewart, R. L. and T. O. T. Ts'o. PDP-8 foreground/background revisited. Digital Equipment Computer User Society, Fall Meeting, San Francisco, November, 1973.

9. Ts'o, T. O. T. A Rapid Method of Segregation of Rat Population for In-depth Psychopharmacological Research. The Pharmacologist 15: 253, 1973.

10. Ts'o, T. O. T., R. L. Stewart and F. J. Karle. A foreground/ background and control system using the PDP-8/i computer in phychopharmacological research. Digital Equipment Computer User Society, Fall Meeting, San Francisco, November, 1969.

11. Thorndike, E. L. Animal intelligence. An experimental study of the associative processes in animals. Psychol. Monogr. 2: pp. 109,1898 .

12. Winer, B. J. Statistical Principles in Experimental Design. New York: McGraw-Hill, 1971, pp. 514-604. 